\title{
Latest developments in the field of stem cell research and regenerative medicine compiled from publicly available information and press releases from nonacademic institutions, 1-30 September 2016
}

First draft submitted: 12 October 2016; Accepted for publication: 13 October 2016; Published online: 30 November 2016

\section{Business development}

\section{Collaborations, partnerships}

\& alliances

\section{Co-development \& commercialization agreement: Cynata \& FUJIFILM}

Cynata Therapeutics (Australia; http:// cynata.com) announced the execution of a term sheet with FUJIFILM Corporation (Japan; www.fujifilmholdings.com) for the development and commercialization of Cynata technology, including Cynata's lead induced pluripotent stem cell (iPSC)-derived therapeutic mesenchymal stem cell (MSC) product, CYP-001. The term sheet anticipates that, under a definitive agreement, Cynata will grant FUJIFILM an option to an exclusive, worldwide license to market and sell CYP-001 for prevention and treatment of graft-versus-host disease (GvHD), as well as an option to negotiate a license for manufacturing those products, and rights to Cynata's proprietary Cymerus ${ }^{\mathrm{TM}}$ technology used to produce MSC precursors, mesenchymoangioblasts, from iPSCs. Under a definitive agreement, FUJIFILM will make a direct investment through the acquisition of Cynata shares to the value of US\$3 million, and paying upfront and milestone payments to Cynata, together with a royalty on end product sales.

\section{Collaboration agreement: Plasticell \& Cellspring}

Plasticell (UK; www.plasticell.co.uk) has announced collaboration with CellSpring (Switzerland; http://cellspring.co/index. html), a company that has developed a high throughput $3 \mathrm{D}$ cell culture system, the 3D Bloom ${ }^{\circledR}$ Biopolymer Platform, which enables more informed 'stop/go' decisions for preclinical candidates. Plasticell and CellSpring are developing tissue models using 3D Bloom Biopolymer seeded with human MSCs that are subsequently differentiated to bone, cartilage and brown/white fat tissue using CombiCult ${ }^{\circledR}$ derived differentiation media. 3D cultures of cells resemble natural tissues more closely compared with conventional 2D cultures grown on flat dishes. In particular, 3D tissues have more accurate biological responses to drug treatment and are used in the pharmaceutical industry to identify promising lead compounds in all stages of drug discovery.

\section{License agreement: GE Healthcare \& STEMCELL Technologies}

GE Healthcare (UK; www.gehealthcare. com) has agreed with STEMCELL Technologies (Canada; www.stemcell.com) to provide T-cell reagents for commercial-scale cell therapy production. Under the agreement, GE Healthcare will commercialize current good manufacturing practice-grade versions of STEMCELL Technologies' T-cell reagents for the isolation, activation and culture of $\mathrm{T}$ cells in clinical applications. These reagents are critical tools in the development and manufacturing of cell and gene therapies intended for administration to patients.
Dusko Ilic ${ }^{*, 1} \&$ Cristina Trento ${ }^{2}$ 'Stem Cell Laboratories, Guy's Assisted Conception Unit, Division of Women's Health, Kings College, London, UK ${ }^{2}$ Department of Haemato-Oncology, Rayne Institute, Faculty of Science \& Medicine, King's College London, London, UK

*Author for correspondence: dusko.ilic@kcl.ac.uk 
Partnership agreement: Fate \& Memorial Sloan Kettering Cancer Center

Fate Therapeutics (CA, USA; www.fatetherapeutics. com) has entered to a partnership with Memorial Sloan Kettering Cancer Center (NY, USA; www.mskcc.org) for the development of off-the-shelf T-cell product candidates using engineered pluripotent cell lines.

\section{Partnership agreement: L'Oreal \& Poietis}

L'Oréal (France; www.loreal.com) and Poietis (France; www.poietis.com) are giving themselves the means to pursue a new scientific challenge: bioprinting a hair follicle, the small organ that produces hair, using a bioprinter. The laser-assisted bioprinting technology developed by Poietis to produce biological tissue can position cells in $3 \mathrm{D}$ with extremely high cellular resolution (on the order of 10 microns) and cellular viability (over 95\%). This unique bioprinting technology involves successively layering microdrops of bioinks using a quick scan by a laser beam. The living biological tissue created must then be matured for around 3 weeks before it can be used in tests. The combination of this exclusive technology with L'Oréal's unique expertise in hair biology could make it possible to create a functional follicle capable of producing hair.

\section{Launching new projects, products \& services Cellenkos}

Golden Meditech Holdings (Hong Kong; www.goldenmeditech.com/eng/global/home.php) and The University of Texas at MD Anderson Cancer Center (TX, USA; www.mdanderson.org) have created Cellenkos, a start-up enterprise focused on umbilical cord blood derived T-reg cellular therapies. Cellenkos, to be based in Houston, is funded with an initial investment of US $\$ 10$ million with warrants to purchase an additional US $\$ 10$ million worth of shares by Golden Meditech and an independent strategic investor. Golden Meditech and the strategic investor will collectively own $51 \%$ of the voting stock of the new company following the exercise of the warrants. The investment includes options to expand the T-reg technologies of Cellenkos into key Asian markets.

\section{Cellular Dynamics International}

Cellular Dynamics International (CDI) (WI, USA; www.cellulardynamics.com), a FUJIFILM (Japan; www.fujifilmholdings.com) company, announced that effective 1 September 2016, the company operations were divided into two equally important business units: Therapeutics Business Unit and The Life Science Business Unit. CDI's new Therapeutics Business Unit will be focused on developing and bringing to market iPSC-based therapies in the ocular, cardiac, Parkin- son's and oncology spaces, with IND applications in all these therapeutic areas expected in the 2018-2020 time period. The Life Science Business Unit will concentrate on building the research products business by propelling innovative cell products, applications and services into the fast growing iPSC marketplace.

In unrelated press release, the company announced the launch of iCell ${ }^{\circledR}$ Hepatoblasts, liver progenitor cells generated from human iPSCs. These cells show promise for liver regeneration therapy research by enabling researchers to identify molecules capable of stimulating liver progenitor cell proliferation and growth. They also provide an in vitro cellular system for studying hepatic development and regeneration and they can be engineered to develop complex liver models for use in in vitro and in vivo applications.

Furthermore, CDI has launched a new venture Opsis Therapeutics. Opsis Therapeutics (WI, USA; http://opsistherapeutics.com) was formed on 31 July and is focused on discovering and developing novel medicines to treat patients suffering from retinal diseases. Therapeutic candidates will be developed in conjunction with the company's haplobanking, enabling the therapeutic cells to be matched to the patient's immune system. CDI has an ongoing contract with the National Eye Institute to manufacture clinically compatible iPSC-derived human retinal pigment epithelial cells.

\section{MiMedx}

MiMedx Group (GA, USA; www.mimedx.com) launched AmnioFill ${ }^{\mathrm{TM}}$ - the first product in the MiMedx placental collagen matrix product family. AmnioFill is a collagenous matrix derived from the placenta and comprised of placental extracellular matrix tissue. AmnioFill is a tissue allograft containing extracellular matrix proteins, growth factors, cytokines and other specialty proteins present in placental tissue. Over 226 growth factors, cytokines and chemokines, including important modulators of inflammation and factors critically important in wound healing, are contained in the AmnioFill placental tissue. MiMedx employs terminal sterilization in addition to aseptic processing techniques in its proprietary processing methodology to enhance the safety of AmnioFill and its other amniotic and placental products.

\section{UKSCB}

The UK Steering Committee has approved 38 UK embryonic stem cell lines for clinical application and the UK Stem Cell Bank (UK; www.nibsc.org/ukstemcellbank) has now executed legal agreements with the Depositors for all these cell lines. Furthermore, the Bank has already accessioned 27 of these and has 
completed its Due Diligence process on 20 cell lines, all of which have been confirmed as The European Union Tissue and Cells Directives Grade - in other words, compliant with the Human Tissue Act Directions and suitable for use as starting materials for

\section{Clinical trials}

\section{Asterias}

Asterias Biotherapeutics (CA, USA; www.asteriasbiotherapeutics.com) presented positive interim efficacy data from the ten million cell cohort, in the ongoing AST-OPC1 SCiSTAR Phase I/IIa multicenter clinical study in complete cervical spinal cord injury (SCI) patients, at the 55th Annual Scientific Meeting of the International Spinal Cord Society in Vienna, Austria. AST-OPC1 is being administered 14-30 days postinjury at up to 20 million cells in 35 patients with subacute, C5-C7, motor complete cervical SCI. Patients are being followed by neurological exams and imaging procedures to assess the safety and activity of the product. Five out of five patients infused with 10 million cells exhibited improved upper extremity motor scores (UEMS) relative to baseline. At day 90 of follow-up, four of four patients dosed improved one motor level on at least one side, two of four patients improved two motor levels on at least one side and one patient improved two motor levels on both sides. The average UEMS improvement at day 90 for the four patients that have reached this follow up was 9.5 points. No serious adverse events related to AST-OPC1, the injection procedure, or immunosuppression with low-dose tacrolimus were found. In addition, data from the study indicate that AST-OPC1 can be safely administered to patients in the subacute period after severe cervical SCI. In the patient cohort treated with two million cells, three out of three patients exhibited improved UEMS relative to baseline, and the average UEMS improvement for the three patients was 5.0 points at day 90, and they continued to improve an average of 7.0 points at 1 year. The company expects to have data evaluating the efficacy results after implantation of 20 million AST-OPC1 cells in complete cervical SCI patients later in 2017. Additional information on the Phase I/IIa trial, including trial sites, can be found at: www.clinicaltrials.gov (ID: NCT02302157), and at the SCiStar Study website (www.scistar-study.com).

\section{Bluebird}

Bluebird Bio (MA, USA; www.bluebirdbio.com) has opened HGB-207, a Phase III, single-arm, global, multicenter study designed to evaluate the safety and efficacy of LentiGlobin BB305 drug product in 15 patients with transfusion-dependent $\beta$-thalassemia (TDT) clinical applications under the EU regulation. Due Diligence on a further seven cell lines is being conducted. Detailed human leukocyte antigen data has been received from Depositors for 34 of the lines and is available upon request.

and non- $\beta 0 / \beta 0$ genotypes (www.clinicaltrials.gov, ID: NCT02906202). The study's primary end point is transfusion independence, defined as a 12-month transfusion-free period after transplant. In this study, the process by which the patient's cells are transduced with LentiGlobin will be modified by the addition of two transduction enhancers during the transduction step of the manufacturing process, intended to increase vector copy number and the percentage of cells successfully transduced. Bluebird also intends to incorporate these transduction enhancers into the manufacturing process for HGB-206, its ongoing study of LentiGlobin in patients with severe sickle cell disease.

\section{Caladrius}

Caladrius Biosciences (NJ, USA; www.caladrius.com) completed enrollment of the first patient cohort in the Phase II Sanford Project: T-Rex study occurred in August as planned. The T-Rex study is investigating Caladrius' product candidate, CLBS03 (autologous expanded Tregs), for the treatment of recent onset Type 1 diabetes. The complete study targets inclusion of 111 patients, by design divided into an initial cohort of approximately 18 patients for an initial safety evaluation followed by a second cohort to meet patient enrollment goals. CLBS03 has received Fast Track and Orphan Drug designations from the US FDA as well as Advanced Therapeutic Medicinal Product classification from the EMA. Additional information on this prospective, randomized, placebo-controlled, doubleblind Phase II clinical trial can be found at www. clinicaltrials.gov (ID: NCT02691247).

\section{Gradalis}

Gradalis (TX, USA; www.gradalisinc.com) announced that the first patient has been dosed in an open label, investigator-sponsored pilot study combining $\mathrm{Vigil}^{\circledR}$ Engineered Autologous Tumor Cells (EATCs) with durvalumab - an investigational human monoclonal antibody directed against PD-L1 - in locally advanced or metastatic triple-negative breast cancer patients that have progressed following two prior lines of therapy. This is supported partly by a grant from Gradalis, to evaluate the safety, tolerability and efficacy of the combination of Vigil and durvalumab. Vigil is an investigational cellular immunotherapy technology that 
combines the concepts of genetic engineering with the science of immunooncology, to enable an immune response to cancer cells. A patient's tumor cells are engineered with a plasmid carrying the gene vector for shRNA Furin and GMCSF to elicit a systemic T-cell directed immune response when administered to the patient through intradermal injections. By utilizing the patient's own tumor as the antigen source, Vigil EATC is designed to elicit an immune response that is specifically targeted and broadly relevant to each patient's unique tumor antigens. Vigil EATC is being studied in Ewing's sarcoma and ovarian cancer as a single agent, and in breast cancer, non-small-cell lung cancer and melanoma in combination with PD-L1 inhibitors. More information about these studies can be found on www.vigilclinicaltrials.com. PD-L1 expression enables tumors to evade detection from the immune system through binding to PD-1 on cytotoxic T lymphocytes. Durvalumab blocks PD-L1 interaction with both PD-1 and CD80 on T cells, countering the tumor's immuneevading tactics. Durvalumab is being investigated in an extensive clinical trial program, as monotherapy or in combination, in non-small-cell lung cancer, bladder, head and neck, gastric, pancreatic, hepatocellular carcinoma and blood cancers.

\section{Juno}

The Fred Hutchinson Cancer Research Center (WA, USA; www.fredhutch.org) published the data from an early-phase study of patients with advanced nonHodgkin lymphoma receiving Juno Therapeutics' (WA, USA; www.junotherapeutics.com) proprietary product, JCAR014, a chimeric antigen receptor (CAR) T-cell treatment, and chemotherapy [1]. JCAR014's hallmark is its use of a one-to-one ratio of helper $\left(\mathrm{CD}^{+}\right)$and killer $\left(\mathrm{CD} 8^{+}\right)$CAR T cells, which are a patient's own immune cells that are genetically engineered to identify and kill cancer cells. The paper reported the results of the first 32 patients in a dose-finding trial of JCAR014 following a round of lymphodepletion, designed to create a more favorable environment for the CAR T cells to grow in the patient's body. Key findings of the study demonstrated the importance of the choice of lymphodepletion regimen and the effects of different doses of CAR T cells. Fifty percent of the 18 patients who were evaluable for efficacy after receiving CAR T cells and chemotherapy agents fludarabine and cyclophosphamide (Cy/Flu) had a complete response, which compares favorably to the $8 \%$ complete response rate in patients who received JCAR014 plus cyclophosphamide-based chemotherapy without fludarabine. Dose-limiting toxicities were observed in some patients in this dosefinding study who received the highest CAR T-cell dose. The study continues with the intermediate CAR T-cell dose. In patients that received Cy/Flu lymphodepletion and the intermediate dose of JCAR014, the data showed a promising early efficacy and side effect profile.

\section{Kerastem}

Kerastem (CA, USA: http://kerastem.com) has completed enrollment of STYLE, the company's US Phase II clinical trial (www.clinicaltrials.gov, ID: NCT02503852). STYLE is a Phase II randomized, double-blind and controlled investigation of Kerastem therapy in early stage female and male pattern baldness. The Kerastem therapy is a combination of fat-based stem and regenerative cells and fat tissue purified with Puregraft (www.puregraft.com). The primary objective of this study is to evaluate the safety and feasibility of the Celution and Puregraft Systems in the processing and preparation of an autologous fat graft enriched with adipose-derived regenerative cells in the treatment of early alopecia androgenetica.

\section{Kite}

Kite Pharma (CA, USA; http://kitepharma.com) announced positive results from an interim analysis of ZUMA-1 for KTE-C19 in patients with chemorefractory diffuse large B-cell lymphoma. KTE-C19 is an investigational therapy in which a patient's $\mathrm{T}$ cells are engineered to express a CAR to target the antigen CD19, a protein expressed on the cell surface of B-cell lymphomas and leukemias, and redirect the $\mathrm{T}$ cells to kill cancer cells. KTE-C19 met the primary end point of objective response rate (ORR), $\mathrm{p}<0.0001$ with an objective response rate of $76 \%$, including $47 \%$ complete remissions. ZUMA-1 enrolled patients with chemorefractory aggressive non-Hodgkin lymphoma into two cohorts. Cohort 1 included patients with diffuse large B-cell lymphoma, and cohort 2 enrolled patients with transformed follicular lymphoma and primary mediastinal B-cell lymphoma. The interim analysis of ZUMA-1 evaluated the ORR in the first 51 patients in cohort 1 with at least 3 months of follow-up. This analysis also included an additional 11 patients in cohort 2. Across the combined 62 patients, the most common grade 3 or higher adverse events included neutropenia (66\%), anemia (40\%), febrile neutropenia (29\%), thrombocytopenia (29\%) and encephalopathy (26\%). Grade 3 or higher cytokine release syndrome and neurological toxicity was observed in 18 and $34 \%$ of patients, respectively. Two patients died from KTE-C19 related adverse events (hemophagocytic lymphohistiocytosis and cardiac arrest in the setting of cytokine release syn- 
drome). The Phase II interim outcomes in ZUMA-1 are largely consistent with results from the Phase I portion of the study and the National Cancer Institute study based on the same CAR construct a low-dose cyclophosphamide-fludarabine conditioning regimen.

\section{SanBio}

SanBio (CA, USA; http://sanbio.com) has begun a Phase II clinical trial of regenerative cell medicine SB623 for patients affected by traumatic brain injury in Japan. SanBio had previously announced the world's first global Phase II clinical trial for chronic traumatic brain injury with allogeneic stem cells, with patient enrollments already begun in the USA. The STEMTRA trial is designed to study the safety and efficacy of SB623 cell therapy in treating patients with chronic motor impairments following traumatic brain injury. The trial will enroll 52 subjects, and Japanese patients will now be included in this trial (www.clinicaltrials. gov, ID: NCT02416492). SB623 cells are modified allogeneic MSCs, derived from bone marrow stromal cells isolated from healthy adult donors, which, administered into neural tissue, should promote recovery from injury by triggering the brain's natural regenerative ability. SanBio recently completed a US-based Phase I/IIa clinical trial for SB623 in patients with chronic motor impairments of 6 months to 5 years following an ischemic stroke (www.clinicaltrials.gov, ID: NCT01287936). Based on these results, a Phase IIb randomized double blind clinical trial of 156 subjects began enrollment in December 2015 for the stroke indication (www.clinicaltrials.gov, ID: NCT02448641). The STEMTRA trial extends the evaluation of SB623 to patients afflicted by traumatic brain injury, which can result in lifelong motor deficits, and for which there are currently no approved medicine.

\section{Regulations, approvals \& acquisitions}

\section{Green light \\ Asterias}

Asterias Biotherapeutics (CA, USA; www.asteriasbiotherapeutics.com) announced that its Data Monitoring Committee has reviewed the safety data from the initial cohort of three patients dosed with 2 million cells, and a subsequent five patients in the second cohort dosed with 10 million cells, and has cleared the company to now begin dosing a third cohort of 5-8 complete cervical injury patients (AIS-A patients) with the highest dose of 20 million cells. Concurrently, the study is also proceeding with enrolling the first cohort of 5-8 sensory incomplete cervical SCI patients (AIS-B patients), each of whom will be administered ten million cells. The SCiStar study is an ongoing Phase I/IIa clinical trial funded in part by a US $\$ 14.3$ million grant from the California Institute for Regenerative Medicine and is designed to evaluate the safety and efficacy of escalating doses of AST-OPC1 in newly injured patients with sensory and motor complete cervical SCI as well as newly injured patients with sensory incomplete SCI. These patients are commonly referred to as AIS-A and AIS-B patients, respectively. Additional information on the Phase I/IIa trial, including trial sites, can be found at www.clinicaltrials.gov (ID: NCT02302157), and at the SCiStar Study website (www.scistar-study. com).

\section{Athersys}

Athersys (OH, USA; www.athersys.com) has received agreement from the FDA under a special protocol assessment for the design and planned analysis of a Phase III Study of MultiStem Treatment for Isch- emic Stroke. The Phase III trial titled, MASTERS2, would provide the foundation of the regulatory package to be submitted for marketing approval. Multistem cell therapy is a patented regenerative medicine product based on stem cells isolated from bone marrow or other tissues, that have the ability to promote tissue repair and healing through the production of therapeutic factors produced in response to signals of inflammation and tissue damage. The MASTERS2 clinical trial will be a randomized, double-blind, placebo-controlled clinical trial designed to enroll 300 patients in North America and Europe who have suffered moderate to moderate-severe ischemic stroke. The enrolled subjects will receive either a single intravenous dose of MultiStem cell therapy or placebo, administered within $18-36 \mathrm{~h}$ of the occurrence of the stroke, in addition to the standard of care. The primary end point will evaluate disability using modified Rankin Scale scores at 3 months, comparing the distribution between the MultiStem treatment and placebo groups. The modified Rankin Scale shift analysis considers disability across the full spectrum, enabling recognition of large and small improvements in disability and differences in mortality and other serious outcomes, among strokes of different severities. The study will also assess excellent outcome (modified Rankin Scale $\leq 1$, NIHSS $\leq 1$ and Barthel Index $\geq 95$ ) at 3 months and 1 year as key secondary end points. Additionally, the study will consider other measures of functional recovery, biomarker data and clinical outcomes, including hospitalization, mortality and life-threatening adverse events, and post-stroke complications such as infection. 
Furthermore, the company announced the successful completion of Japan's Pharmaceutical and Medical Devices Agency review of the Clinical Trial Notification, allowing the commencement by Healios (Japan, www.healios.co.jp/en) of a confirmatory clinical trial evaluating the safety and efficacy of administration of MultiStem for the treatment of ischemic stroke in Japan (also designated by Healios as HLCM051 in Japan). In accordance with the regulatory system in Japan, a Clinical Trial Notification is equivalent to an Investigational New Drug (IND) application under the FDA. The clinical trial to be conducted in Japan stems from a partnership and license agreement between Healios and Athersys, and consists in a randomized, double-blind, placebo-controlled clinical trial where subjects will receive either a single dose of MultiStem or placebo, administered within 18-36 h of the occurrence of the stroke, in addition to standard of care. The study will evaluate patient recovery through approximately 90 days following initial treatment based on excellent outcome and other neurological, functional and clinical end points. The trial in Japan follows a Phase II study, B01-02 trial, which was completed at 33 clinical sites in the USA and UK, and evaluated safety and effectiveness of the intravenous administration of MultiStem cells within 24-48 h after the occurrence of a moderate-to-severe stroke on 126 subjects.

\section{Bluebird}

The EMA has granted access to its Priority Medicines scheme for Bluebird Bio's (MA, USA; www. bluebirdbio.com) proprietary product LentiGlobin in the treatment of patients with TDT. The Priority Medicines initiative provides enhanced support and increased interaction to companies, with the goal of optimizing development plans and speeding regulatory evaluations to bring innovative medicines to patients more quickly. Bluebird Bio is also participating in the EMA's Adaptive Pathways Pilot program, which also aims to expedite patient access to new therapies. LentiGlobin BB305 product aims to treat TDT and severe sickle cell disease (SCD), by inserting ex vivo a functional human $\beta$-globin gene into a patient's own hematopoietic stem cells outside the body and then transplanting those modified cells into the patient's blood stream. Promising results from a preclinical proof-of-concept study using gene therapy to treat SCD were published earlier [2]. Bluebird Bio has three ongoing studies evaluating LentiGlobin therapy for the treatment of TDT and severe SCD - the Northstar Study in TDT, the HGB-205 study in TDT or severe SCD and the HGB-206 study in severe SCD.
Cynata

Cynata Therapeutics (Australia, http://cynata.com) has received approval from the UK Medicines and Healthcare products Regulatory Agency to proceed with its Phase I clinical trial of CYP-001 in patients with steroid resistant GvHD. CYP-001 is Cynata's lead Cymerus MSC product, developed through the Cymerus technology utilizing iPSC and mesenchymoangioblasts to achieve economic manufacture of MSC at commercial scale. Cynata plans to conduct the Phase I clinical trial, which is titled "An Open-Label Phase I Study to Investigate the Safety and Efficacy of CYP-001 for the Treatment of Adults With SteroidResistant Acute Graft Versus Host Disease”, at a number of leading clinical centers in the UK and Australia. Additional centers in other jurisdictions are also being considered. The trial will aim to recruit approximately 16 participants who have undergone a bone marrow transplant or similar procedure, and were subsequently diagnosed with steroid resistant grade II-IV acute GvHD.

\section{Fate}

The FDA has granted Orphan Drug Designation to Fate Therapeutics (CA, USA; www.fatetherapeutics. com) for ProTmune ${ }^{\mathrm{TM}}$. The FDA designation is for "prevention of GvHD in patients undergoing allogeneic hematopoietic cell transplantation" and broadly covers diseases, including blood cancers and genetic disorders, for which the procedure is performed. ProTmune ${ }^{\mathrm{TM}}$ is an investigational programmed cellular immunotherapy undergoing clinical development for the prevention of acute GvHD and cytomegalovirus infection in patients undergoing allogeneic HCT. The cell therapy is produced by modulating a donorsourced, mobilized peripheral blood graft ex vivo with two small molecules (FT1050 and FT4145) to enhance the biological properties and therapeutic function of the graft's immune cells. The programmed mobilized peripheral blood graft is administered to a patient as a one-time intravenous infusion.

\section{Pending}

\section{RegenBio}

Regen BioPharma (CA, USA; www.regenbiopharmainc.com) submitted a revised IND application to the FDA, which will be focusing on using a novel method to unleash the body's innate ability to kill cancer. The package submitted contained preclinical experiments as well as details of the proposed clinical trial, and proposes to combine the company's proprietary dCellVax product with silencing through siRNA of Indoleamine 2,3-dioxygenase, an enzyme that is overexpressed in some cancers and which sup- 
presses immune system response against the cancer. The proposed dCellVax clinical trial involves generation of patient-specific dendritic cells that are modified by gene-silencing to lose expression of the immune checkpoint gene Indoleamine 2,3-dioxygenase. Ten patients with advanced breast cancer will be treated in the proposed clinical trial. The company has also submitted additional data to the FDA supporting its application requesting Orphan Drug

\section{Capital market \& finances}

\section{AiVita}

AiVita Biomedical (CA, USA; www.aivitabiomedical.com) has been awarded a Research Project Grant (R01) from the National Eye Institute of the $\mathrm{NIH}$ for the development of stem cell-derived 3D-transplantable retinas to treat vision loss. The project aims to address the loss of retinal pigment epithelium and photoreceptors typically observed in patients with advanced degenerative eye disease, such as macular degeneration and retinitis pigmentosa. AiVita will manufacture the 3D-retinal organoids and render the process both clinically and commercially compliant, and collaborators at the University of California at Irvine's Sue \& Bill Gross Stem Cell Research Center will test the product for safety and efficacy in relevant models of retinal degeneration.

\section{Allele}

Allele Biotechnology and Pharmaceuticals (CA, USA; www.allelebiotech.com) has been awarded a Phase I Small Business Innovation Research grant to develop a novel manufacturing system to produce stem cell-derived human tissue and cells for clinical therapy from the NIH's National Heart, Lung and Blood Institute. This grant will allow Allele to further move its patented method of reprogramming somatic cells into iPSCs into commercially viable clinical cell therapies.

\section{Capricor}

Capricor Therapeutics (CA, USA; http://capricor. com) has been awarded a grant of approximately US\$2.4 million from the US Department of Defense toward establishing a scalable, commercially ready process to manufacture CAP2003 (CardiosphereDerived Cell Exosomes). Capricor has identified ocular GvHD as the first clinical development opportunity for CAP2003, and expects to submit an IND for this indication in the first half of 2017. CAP2003 represents exosomes isolated from the company's proprietary cardiosphere-derived cells [3] which are currently in clinical evaluation by Capricor for the status for the use of HemaXellerate in aplastic anemia. HemaXellerate is comprised of cells extracted from the patient's own fat tissue and processed using a proprietary method to induce a biological response in the patient that heals damaged bone marrow and restores ability of the body to generate healthy blood cells. The FDA recently cleared Regen to perform Phase I clinical trials using HemaXellerate in aplastic anemia patients.

treatment of several cardiac conditions, including that associated with Duchenne muscular dystrophy (www.clinicaltrials.gov, ID: NCT02485938).

\section{Cytori}

Cytori Therapeutics (CA, USA; www.cytori.com) announced that the Biomedical Advanced Research and Development Authority (BARDA; www.phe. gov/about/BARDA/Pages/default.aspx), a division of the US Department of Health and Human Services, increased the contract option originally signed in August 2014 to fund continued investigation and development of Cytori Cell Therapy for use in thermal burn injuries. Cytori Cell Therapy is based on the use of adipose derived regenerative cells isolated from a patient's own adipose tissue. The amended contract option is valued at US\$16.6 million, an increase of approximately US $\$ 2.5$ million from its previous value of US\$14.1 million. Upon Investigational Device Exemption approval by the FDA, if received, Cytori will request that BARDA provide additional funding to cover costs associated with the completion of a pilot clinical trial. This trial will employ in vivo administration of Cytori Cell Therapy. The supplemental funds from this amended contract will be used to support the remaining activities necessary to seek approval of the Investigational Device Exemption and support clinical readiness. The original contract includes additional options, exercisable at BARDA's discretion, valued at up to US\$68 million to fund pilot clinical trials and additional work in thermal burn complicated by radiation exposure.

\section{Financial \& competing interests disclosure}

The authors have no relevant affiliations or financial involvement with any organization or entity with a financial interest in or financial conflict with the subject matter or materials discussed in the manuscript. This includes employment, consultancies, honoraria, stock ownership or options, expert testimony, grants or patents received or pending, or royalties.

No writing assistance was utilized in the production of this manuscript. 


\section{References}

1 Turtle CJ, Hanafi LA, Berger C et al. Immunotherapy of non-Hodgkin's lymphoma with a defined ratio of $\mathrm{CD}^{+}$and $\mathrm{CD} 4{ }^{+} \mathrm{CD} 19$-specific chimeric antigen receptor-modified T cells. Sci. Transl.Med. 8(355), 8:355ra116 (2016).
2 Pawliuk R, Westerman KA, Fabry ME et al. Correction of sickle cell disease in transgenic mouse models by gene therapy. Science 294(5550), 2368-2371 (2001).

Ibrahim AG, Cheng K, Marbán E. Exosomes as critical agents of cardiac regeneration triggered by cell therapy. Stem Cell Reports 2(5), 606-619 (2014). 